\title{
Téoros
}

Revue de recherche en tourisme

\section{Changement climatique, authenticité et marketing des régions nordiques}

\author{
Conséquences sur le tourisme finlandais et la «plus grande \\ marque au monde " ou « Les changements climatiques \\ finiront-ils par tuer le père Noël ?"
}

\section{Michael Hall}

Volume 28, numéro 1, 2009

Tourisme polaire

URI : https://id.erudit.org/iderudit/1024838ar

DOI : https://doi.org/10.7202/1024838ar

Aller au sommaire du numéro

Éditeur(s)

Université du Québec à Montréal

ISSN

0712-8657 (imprimé)

1923-2705 (numérique)

Découvrir la revue

Citer cet article

Hall, C. M. (2009). Changement climatique, authenticité et marketing des régions nordiques : conséquences sur le tourisme finlandais et la " plus grande marque au monde " ou " Les changements climatiques finiront-ils par tuer le père Noël ? ». Téoros, 28(1), 70-79. https://doi.org/10.7202/1024838ar

\section{Résumé de l'article}

Cet article expose le rôle du père Noël comme élément central du marketing et de la compétitivité des hautes latitudes nordiques. Bien que souvent associé à la Finlande et à la Laponie, nous démontrons qu'un certain nombre de destinations tendent à utiliser le père Noël dans leur effort de développer un avantage construit. Nous soutenons par ailleurs que la valeur du père Noël comme moyen de différencier une destination s'amoindrira à court terme en raison de la perte d'authenticité qui découle de la concurrence d'autres destinations et peut-être davantage à long terme comme une des conséquences du changement climatique. 


\title{
Changement climatique, authenticité et marketing des régions nordiques Conséquences sur le tourisme finlandais et la «plus grande marque au monde» ou «Les changements climatiques finiront-ils par tuer le père Noël ${ }^{1}$ ?»
}

\author{
C. Michael HALL, Ph.D. \\ Professeur \\ Université de Canterbury (Nouvelle-Zélande) \\ michael.hall@canterbury.ac.nz
}

RÉSUMÉ : Cet article expose le rôle du père Noël comme élément central du marketing et de la compétitivité des hautes latitudes nordiques. Bien que souvent associé à la Finlande et à la Laponie, nous démontrons qu'un certain nombre de destinations tendent à utiliser le père Noël dans leur effort de développer un avantage construit. Nous soutenons par ailleurs que la valeur du père Noël comme moyen de différencier une destination s'amoindrira à court terme en raison de la perte d'authenticité qui découle de la concurrence d'autres destinations et peut-être davantage à long terme comme une des conséquences du changement climatique.

Mots-clés : Stratégie de promotion régionale, changements climatiques, Noël, authenticité, avantages construits.

Le marketing est une dimension clé en tourisme puisqu'il constitue un des liens qui existent entre le désir et la demande latents $\mathrm{du}$ consommateur et leur conversion en expérience concrète de consommation du produit touristique. De nombreuses régions tentent de faire leur propre marketing en développant une image de destination forte qui les différencie des autres destinations dans un marché très concurrentiel eu égard à leur image. Parce qu'il est souvent utilisé pour impulser le redéveloppement, la revitalisation et les stratégies de promotion régionaux, le tourisme est étroitement lié au processus de marketing de la destination. On admet par ailleurs de plus en plus que le marketing des destinations touristiques est interrelié avec d'autres formes de concurrence et de promotion de la destination. Le marketing de la destination, aussi appelé branding ou image de la destination, ou encore le marketing ou l'image de la marque (Hall, 1997; Page et Hall, 2003; Malecki, 2004; Jansson et Power, 2006), fait maintenant partie intégrante non seulement de la façon dont la destination séduit le touriste, mais également de la façon d'attirer et de retenir d'autres éléments mobiles, notamment les investissements, les entreprises et les gens, dans un effort de promouvoir le développement économique régional (Kotler et al., 1999; Kotler et Gertner, 2002). Bien que la valeur des stratégies de compétitivité de la destination soit toujours fortement contestée, nombre de décideurs politiques ont développé une passion pour le discours autour de la compétitivité de la destination (Malecki, 2004; Bristow,
2005; Hall, 2005; 2007; Nyseth et Granås, 2007). Notre objectif dans le présent article est d'examiner le marketing et la compétitivité de la destination dans un contexte de haute latitude, mais particulièrement en ce qui touche une des plus grandes icônes des régions polaires, le père Noël. Nous abordons aussi certaines des questions qui entourent l'utilisation du père Noël en tant que marque, mais surtout eu égard à l'authenticité et aux changements climatiques.

\section{Marketing et compétitivité de la destination}

Bien que traditionnellement davantage associés à la concurrence entre des sites urbains pour être reconnus comme "ville mondiale», les centres de haute altitude s'engagent de plus en plus sur la voie de la compétitivité de la destination, que ce soit pour faire eux-mêmes leur promotion à titre de porte d'entrée du pôle Nord ou pour attirer l'industrie et les investissements ou davantage de touristes (voir entre autres Hall, 2000). En effet, la perspective « cosmopolite» de la plupart des recherches sur le marketing de la destination, même dans un contexte nordique (Jansson et Power, 2006; Hall et al., 2009), est teintée d'ironie car on peut avancer, en termes comparatifs, que les régions en périphérie ont grandement besoin des avantages qui découlent de nouveaux capitaux, entreprises et personnes (Müller et Jansson, 2007).

Le discours sur la compétitivité de la destination urbaine a surtout touché des thèmes de développement régional tels 
que pôles de croissance, technopoles, villes culturelles, villes novatrices, revitalisation, régénération, réseaux, clustering et innovation (Malecki, 2004; Hall et Williams, 2008). Or, à l'exception des trois derniers concepts, ce discours ne s'applique pas vraiment aux régions périphériques en raison des caractéristiques inhérentes à ces dernières, par exemple l'accès difficile et une population relativement clairsemée. Cela ne signifie pourtant pas que ces régions ne peuvent être créatrices ou culturelles, mais suggère plutôt que, en comparaison avec les grands centres urbains, de telles notions politiques peuvent être difficiles à mettre en œuvre en termes compétitifs. Cependant, la pensée politique régionale contemporaine est bien pertinente aux régions nordiques de haute altitude, surtout si l'on considère que le concept d'avantages régionaux, traditionnellement axé sur les avantages concurrentiels ou comparatifs, met maintenant l'accent sur les avantages construits entre les régions (Cooke et Leydesdorff, 2006).

La notion d'avantage construit est essentielle pour identifier le rôle clé que le marketing et les pratiques de compétition de la destination qui y sont associées, y compris le branding ou l'image de marque, peuvent jouer. Arès tout, la marque de la destination n'est-elle pas une tentative d'imprimer l'avantage de la destination dans l'esprit des consommateurs? Cette notion est très évidente dans la définition de l'American Marketing Association (2008) : «A name, term, design, symbol, or any other feature that identifies one seller's good or service as distinct from those of other sellers.» L'image de la destination est donc le développement et la promotion de la marque d'une destination dans le but de la différencier des autres et ainsi d'accumuler des avantages pour ses compagnies, ses organisations, son peuple, ses produits et services (Hall, 2008).

Le marketing de la destination et le branding résultent d'un éventail de moyens matériels et immatériels. Les stratégies matérielles comprennent des mécanismes tels que des projets phares (centre des congrès, nouvel hôtel ou aéroport, souvent inclus dans de grandes stratégies de développement régional). Les stratégies immatérielles comprennent l'utilisation de publicités, de slogans, des annonces dans les médias et le développement de nouveaux mythes sur la destination (Jansson et Power, 2006). Les projets phares sont souvent plus difficiles à réaliser dans les régions périphériques en raison du manque relatif de capitaux, mais certains réussissent à en obtenir, comme en témoigne le développement de l'hôtel de glace à Jukkasjärvi, Suède, qui s'est révélé un meilleur élément de branding pour la région en termes de couverture médiatique que la mine voisine de Kiruna (Hall et al., 2009), bien que cette dernière soit plus importante sur le plan économique.

Une stratégie alternative de compétition de la destination, pour plusieurs régions périphériques, pourrait donc être de bâtir leur image sur leurs caractéristiques naturelles (Jacobsen, 2009). Par exemple, Finnmark en Norvège utilise beaucoup cap Nord comme élément phare «socialement construit» de son image. Une autre caractéristique naturelle qui peut être utilisée est un emblème (une plante ou un animal iconique ou charismatique). Par exemple Churchill, au bord de la baie d'Hudson au Manitoba (Canada), se promeut en tant que capitale mondiale de l'ours polaire, alors qu'Umeå en Suède se dit la ville des bouleaux. Or, bien que l' "avantage du premier» soit important pour le marketing et la promotion d'une destination afin que les consommateurs fassent facilement cette association dans leur esprit, il est important de planifier à long terme puisqu'il y a toujours la possibilité que d'autres destinations se positionnent de façon similaire, venant ainsi réduire la valeur d'unicité qui aurait différencié la destination par rapport aux autres.

Il est bien sûr difficile de dupliquer une marque «matérielle» associée à une destination géographique exceptionnelle, par exemple cap Nord, mais il en va tout autrement de l'utilisation de plantes ou d'animaux, sauf s'ils sont extrêmement rares. De même, les infrastructures physiques, notamment un hôtel de glace, peuvent être copiées (Page et Hall, 2003; Malecki, 2004). L'hôtel de glace de Jukkasjärvi, qui date des années 1990, a maintenant des concurrents : l'hôtel de neige de Kirkenes (Norvège, première saison 2006), l'hôtel Igloo Alta (Norvège, première saison 2000), l'hôtel de glace de Duchesnay au Québec (Canada, première saison 2000) et l'hôtel de neige et de glace de Laino (Laponie, première saison 2000); la Finlande, la Norvège et maintenant la Roumaine offrent également des produits similaires. Vu la possibilité de copier des caractéristiques utilisées dans les stratégies de marque, il n'est certes pas surprenant que certaines destinations nordiques se soient tournées vers l'immatériel et l'intangible, souvent par le développement de nouveaux mythes et slogans liés à la destination.

\section{La plus grande marque au monde}

Le père Noël (aussi appelé saint Nicolas et Santa Claus) a été décrit comme la plus grande marque au monde (Arruda, 2003; voir aussi Aaltonen, 2004, pour un commentaire paru dans les médias finlandais). Dans les mots de William Arruda (2003), "Santa Claus is the envy of brand managers everywhere. His brand attributes are clear and desirable to virtually everyone. Even parts of the world that have no connection to the holiday know who he is and what he stands for.» Avec l'ours polaire (Slocum, 2004) et le renne (Hukkinen et al., 2006), le père Noël est un symbole évocateur de la région arctique et du pôle Nord dans la plupart des parties du monde (Hall et al., 2009). La présence du père Noël dans les centres commerciaux, les magasins de détail et les maisons à la grandeur presque des pays développés, d'octobre à décembre, fournit par ailleurs un fort renforcement visuel à la marque et son association avec la fête de Noël encourage manifestement la consommation, l'échange de cadeaux, les dépenses et la connectivité émotionnelle avec la marque, comme partie intégrante de ce qui est appelé l' " esprit de Noël» (Clarke, 2006; 2007). À preuve, près de 744 millions de cartes de Noël ont été envoyées au Royaume-Uni en 2005 (Cards For Good Causes, 2007). De même, aux États-Unis, 1,9 milliard de cartes de Noël sont données chaque année et, en 2002, 20,8 millions d'arbres de Noël destinés à la vente ont été coupés sur des fermes (US Census Bureau, 2005).

Pourtant, bien qu'il y ait une documentation abondante sur le père Noël en tant que marque (Otnes et al., 1994; O'Cass et Clarke, 2002; Pine et Nash, 2002; Snellman, 2006a; Pine et al., 2007 ; Coca-Stefaniak et al., 2008), il y a peu de débats scientifiques concernant la contribution du père Noël sur le plan de la compétitivité des destinations de latitude Nord et sur les 


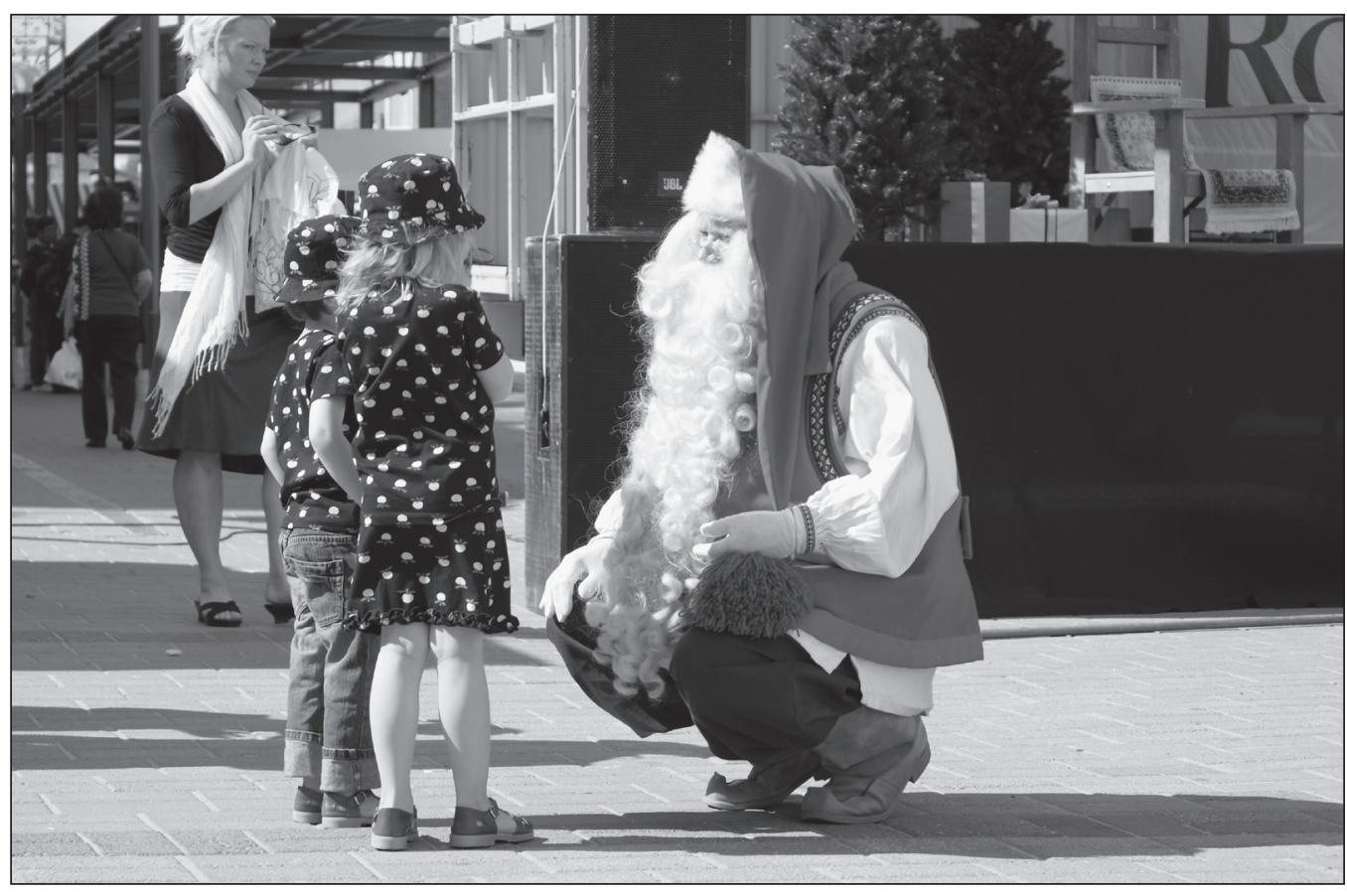

ILLUSTRATION 1 :

Le père Noël (Joulupukki) joue un rôle important pour commercialiser Rovaniemi, la ville autoproclamée capitale de Noël et résidence du célèbre personnage (photo : Alain A. Grenier). façons dont les différentes destinations ont cherché à s'approprier ce personnage (Hall, 2008). S’appuyant sur le développement du village du père Noël à Rovaniemi dans le nord de la Finlande, Michael Pretes (1995) a notamment analysé le développement de l' «industrie du père Noël» en tant que forme de tourisme postmoderne. Bien qu'il soutienne qu'un élément essential de la stratégie de Rovaniemi était de convaincre «the rest of the world that Finland, and Lapland in particular, was the real home of Santa Claus - against rival claimants in Alaska, Sweden, Norway, and Greenland», il n'aborde pas les diverses utilisations du père Noël en tant que partie intégrante des stratégies de compétition.

La section suivante analyse les différentes façons dont les destinations utilisent le père Noël pour faire leur promotion ainsi que quelques-uns des défis qu'elles doivent relever.

\section{Le père Noël et l'image de la destination}

Le tableau 1 présente des exemples de la façon dont le père Noël ou les mythes qui y sont associés sont utilisés pour bâtir l'image de la destination. Bien que cette liste ne soit pas exhaustive, soulignons qu'elle offre quelques-uns des exemples les plus probants où le père Noël est associé à la destination dans le but de promouvoir cette dernière et de se tailler une place parmi les concurrents. Tous les exemples cités sont situés dans des latitudes boréales polaires, à l'exception de Santa Claus, Indiana (États-Unis), qui y paraît en raison de sa désignation postale. Le tableau ne fait donc pas état de parcs d'attractions, comme le Santa's Workshop, The North Pole (Cascade au Colorado) - qui ne mentionne pas le père Noël dans l'image de la destination de juridiction locale (Pikes Peak) -, ni des villages de Demre et de Patara en Anatolie (Turquie), qui ont été des résidences de l'évêque de Myra - source historique de saint Nicolas -, où des agences de marketing de la destination pourraient potentiellement utiliser le père Noël pour promouvoir la localité.

\section{Le père Noël et l'image de la Finlande} et de la Laponie

Ritva Liisa Snellman (2006b) a qualifié la Laponie de Santa tourism superpower en raison du battage considérable fait autour de ce personnage en tant que marque régionale. Bien que l'équivalent finnois du père Noël, ou Joulupukki (bouc de Noël) comme disent les Finlandais, ait longtemps été un personnage de leur folklore, ce n'est que vers la fin des années 1920 qu'on a associé Joulupukki à la montagne de Korvatunturi dans ce qui est connu aujourd'hui comme le parc national Urho Kekkonen en Laponie, sur la frontière finno-russe. Et c'est grâce à une émission de la radio-télévision d'État, qui est restée en ondes jusque dans les années 1950. Plus récemment, la relation entre la Laponie et le père Noël a été renforcée par d'autres médias, plus particulièrement à la suite de la diffusion d'un long métrage intitulé Joulutarina (L'histoire de Noël) (Hytönen, 2007).

L'utilisation du Joulupukki / père Noël pour commercialiser la destination se concentrait initialement sur Rovaniemi (illustration 1), la ville qui s'est autoproclamée capitale de Noël du monde et résidence du père Noël, dont les parcs d'attractions et les activités comportent la plus grande concentration d'infrastructures associées au père Noël et à Noël et qui utilise le plus manifestement le père Noël dans sa stratégie de marque. Néanmoins, le tourisme lié au père Noël est devenu une importante activité touristique pendant la période de Noël pour bon nombre d'autres destinations en Finlande. De telles initiatives régionales ont été impulsées à l'échelle nationale par la Finlande qui se présente comme LE pays de Noël. Par exemple, dès avril 2008, Visit Finland, organisme 
TABLEAU 1 : Exemples d'images de destination associées au père Noël

\begin{tabular}{|c|c|c|c|c|c|}
\hline Pays & Destination & Site(s) Internet & $\begin{array}{l}\text { Personnage / } \\
\text { marque de Noël }\end{array}$ & $\begin{array}{l}\text { Message de la destination / Symbole } \\
\text { immatériel de la marque }\end{array}$ & Symbole matériel de la marque \\
\hline Canada & $\begin{array}{l}\text { Pôle Nord } \mathrm{HOH} \mathrm{OHO} \\
\text { (code postal officiel } \\
\text { pour le courrier } \\
\text { postal envoyé } \\
\text { au père Noël } \\
\text { au Canada) }\end{array}$ & $\begin{array}{l}\text { [canadapost.ca/santascorner] } \\
\text { [http://www.canadapost.ca/dec/santa/ } \\
\text { default-f.asp] }\end{array}$ & $\begin{array}{l}\text { Père Noël } \\
\text { (en français); } \\
\text { Santa Claus } \\
\text { (en anglais) }\end{array}$ & Pôle Nord. & $\begin{array}{l}\text { Au-delà de } 11000 \text { employés actifs et } \\
\text { retraités de Postes Canada, affectueusement } \\
\text { surnommés les lutins des postes, répondent à } \\
\text { plus de un million de lettres et plus de } 40000 \\
\text { courriels (Canada Post, 2007). }\end{array}$ \\
\hline Finlande & Rovaneimi & $\begin{array}{l}\text { [http://www.posti.fi/postimerkkikeskus/ } \\
\text { jpp/en_index.html] (Bureau de poste } \\
\text { principal du père Noël) } \\
\text { [http://www.santaclauslive.com/] } \\
\text { [http://tourism.rovaniemi. } \\
\text { fi/?deptid=6335] (Service } \\
\text { d'information touristique) } \\
\text { [http://www.santaclaus. } \\
\text { fi/?deptid=14561] (Un conte de Noël) } \\
\text { (Rovaniemi Development Agency) }\end{array}$ & $\begin{array}{l}\text { Père Noël; } \\
\text { Rovaneimi, } \\
\text { la ville de noël, } \\
\text { la capitale de Noël }\end{array}$ & $\begin{array}{l}\text { La capitale de la Laponie, Rovaniemi, est une } \\
\text { destination unique tant par sa localisation que } \\
\text { ses services. Située à l'entrée de la Laponie on } \\
\text { peut y traverser le Cercle Arctique, rencontrer } \\
\text { le père Noël et profiter de la luminosité des } \\
\text { nuits estivales ou, en hiver, admirer les aurores } \\
\text { boréales. Rovaniemi constitue une aventure en } \\
\text { elle-même. } \\
\text { C'est au Cercle Arctique que se trouvent le } \\
\text { père Noël et ses lutins; c'est là qu'ils sortent } \\
\text { pour rencontrer les gens. C'est là que la magie } \\
\text { de Noël prend forme. }\end{array}$ & $\begin{array}{l}\text { Le bureau de poste principal du père Noël } \\
\text { reçoit plus de un demi-million de lettres par } \\
\text { année (le site Internet de Tourisme Rovaniemi } \\
\text { en annonce } 700 \text { 000); le parc du père Noël; } \\
\text { le village du père Noël. }\end{array}$ \\
\hline Finlande & Turku & $\begin{array}{l}\text { [http://www.christmascity.com/] } \\
\text { (Bureau of Cultural Affairs, Turku) }\end{array}$ & $\begin{array}{l}\text { Ville de Noël } \\
\text { finlandaise }\end{array}$ & $\begin{array}{l}\text { L'ambiance authentique de la ville de Noël de } \\
\text { Turku est basée sur les longues traditions de la } \\
\text { ville. Turku, la ville finlandaise de Noël, rayonne } \\
\text { de chaleur et de traditions, à partir de la fin } \\
\text { novembre presque jusqu'à la fin de janvier. }\end{array}$ & La rue Christmas, les activités du festival. \\
\hline Groenland & $\begin{array}{l}\text { Le pôle Nord } \\
\text { (avec le Groenland } \\
\text { comme porte } \\
\text { d'entrée) }\end{array}$ & $\begin{array}{l}\text { [http://www.Groenland.com/] } \\
\text { (Sites officiels de tourisme } \\
\text { et d'affaires du Groenland) } \\
\text { [http://www.santa.gl/] }\end{array}$ & Le père Noël & $\begin{array}{l}\text { Peu importe la légende du père Noël en } \\
\text { laquelle vous croyez, il y a une seule chose que } \\
\text { vous devez absolument savoir : le père Noël vit } \\
\text { au Groenland. }\end{array}$ & $\begin{array}{l}\text { Nuuk a un bureau de poste et une boîte postale } \\
\text { consacrés au Père Noël ( } 50000 \text { lettres par } \\
\text { année). }\end{array}$ \\
\hline Islande & $\begin{array}{l}\text { Dimmuborgir, } \\
\text { Myvatnssveit }\end{array}$ & [http://www.santaworld.is/] & $\begin{array}{l}\text { Le père Noël, } \\
\text { les personnages } \\
\text { de Noël }\end{array}$ & $\begin{array}{l}\text { Depuis des temps immémoriaux, le père Noël } \\
\text { a toujours vécu à Dimmuborgir, Myvatnssveit, } \\
\text { où il vaque à son travail qui est de réjouir les } \\
\text { jeunes cœurs, peu importe leur âge, pendant } \\
\text { la période de Noël. Depuis plusieurs années } \\
\text { maintenant le père Noël répond aux lettres des } \\
\text { enfants, laisse des cadeaux dans leur bas de } \\
\text { Noël et distribue des présents à Noël. II réalise } \\
\text { tout cela de chez lui, Dimmuborgir. }\end{array}$ & $\begin{array}{l}\text { Le marché de Noël, le bureau de poste du père } \\
\text { Noël, le Père Noël dans les maisons dès la fin } \\
\text { novembre. }\end{array}$ \\
\hline Norvège & Drøbak & $\begin{array}{l}\text { [http://www.julehus.no/sak/000003. } \\
\text { asp] (Maison de Noël de Tregaarden) }\end{array}$ & $\begin{array}{l}\text { Julenissen } \\
\text { (lutin de Noël) } \\
\text { ou nisser }\end{array}$ & & $\begin{array}{l}\text { Julehuset (la maison de Noël) (10 mois par } \\
\text { année), le bureau de poste (20 } 000 \text { lettres } \\
\text { par année). }\end{array}$ \\
\hline Norvège & Savalen & $\begin{array}{l}\text { [http://www.julenissen.no/barnnisse. } \\
\text { html] }\end{array}$ & $\begin{array}{l}\text { Julenissen } \\
\text { (lutin de Noël) } \\
\text { ou nisser (gnome) }\end{array}$ & & $\begin{array}{l}\text { La rue Santa, la maison et l'atelier du père Noël } \\
\text { (en saison); visites au départ du Royaume-Uni } \\
\text { avec activités outre-mer. }\end{array}$ \\
\hline Suède & Mora (Dalarna) & [http://www.santaworld.se/] & Le père Noël & & $\begin{array}{l}\text { Le monde du père Noël - Tomteland (toute } \\
\text { l'année). }\end{array}$ \\
\hline États-Unis & $\begin{array}{l}\text { Pôle nord, Alaska } \\
\text { (près de Fairbanks) }\end{array}$ & $\begin{array}{l}\text { [http://www.northpolealaska.com/] } \\
\text { [http://www.northpolealaska.com/ } \\
\text { Theme-City/Welcome.html] } \\
\text { [http://www.santaclaushouse.com/] }\end{array}$ & & $\begin{array}{l}\text { Là où l'esprit de Noël est présent toute l'année. } \\
\text { Le père Noël apparaît aussi sur l'emblème } \\
\text { de la ville. } \\
\text { Lorsque développée entièrement comme } \\
\text { une ville à thème, nous pourrons dire avec } \\
\text { enthousiasme : «Le père Noël a préparé une } \\
\text { cité secrète, une place romantique, qui brille } \\
\text { toute l'année des lumières et de la magie de } \\
\text { Noël. Venez visiter le pôle Nord, Alaska!» }\end{array}$ & $\begin{array}{l}\text { La maison du père Noël, une statue du père } \\
\text { Noël de } 12 \text { mètres, le terrain de camping pour } \\
\text { véhicules récréatifs Santaland, la launderette } \\
\text { Santa's Suds, le bureau de poste (400 } 000 \\
\text { lettres par année), la signalisation dans les } \\
\text { rues, représentent tous un thème de Noël. }\end{array}$ \\
\hline États-Unis & Santa Claus, Indiana & $\begin{array}{l}\text { [http://www.santaclausmuseum.org/] } \\
\text { [http://www.legendaryplaces.org/] } \\
\text { (Spencer County Visitor's Bureau } \\
\text { et liens vers le bureau de poste } \\
\text { et les autres attraits) }\end{array}$ & $\begin{array}{l}\text { Le père Noël } \\
\text { (et Abraham } \\
\text { Lincoln) }\end{array}$ & $\begin{array}{l}\text { Aucun conte mythologique sur la destination } \\
\text { de Noël; on présente plutôt un compte rendu } \\
\text { historique : «Venez explorer l'histoire de } \\
\text { notre ville magique; une place extraordinaire } \\
\text { à visiter, où l'entrée est libre» (le musée du } \\
\text { père Noël). } \\
\text { Le bureau de poste du père Noël est situé } \\
\text { sur la Kringle Plaza. }\end{array}$ & $\begin{array}{l}\text { La route principale qui traverse la ville s'appelle } \\
\text { le boulevard Christmas; le château en bonbons } \\
\text { du père Noël, le musée du père Noël; le Holiday } \\
\text { World; la maison du père Noël; le terrain de } \\
\text { camping Lake Rudolph; l'enclave protégée } \\
\text { thématique du village de Christmas Lake; le } \\
\text { bureau de poste; des bénévoles s'assurent que } \\
\text { le père Noël réponde à chaque enfant qui lui } \\
\text { écrit (10 } 000 \text { lettres par année). }\end{array}$ \\
\hline
\end{tabular}


touristique national, rapportait qu'il y avait pour le seul marché du Royaume-Uni 20 voyagistes qui offraient des forfaits Noël en Laponie avec des vols à destination d'Ivalo, de Kajaani, de Kittilä, de Kuusamo et de Rovaniemi, sans compter que l'accès par avion était aussi offert dans des forfaits au départ de Kirkenes (Norvège) et de Kiruna (Suède), qui possèdent des hôtels de glace (Visit Finland, 2008). Le tourisme autour du Père Noël a certes considérablement contribué au développement économique (Lähteenmäki, 2006), le tourisme étant le plus grand contributeur au produit intérieur brut (PIB) pour toutes les régions finlandaises à l'exception d'Åland (Hall et al., 2009). Nigel Halpern (2008) estime par ailleurs que le tourisme international venu en Laponie en avion a contribué 36 millions d'euros à l'économie régionale en 2005 et que pendant la saison des fêtes la majorité des touristes arrivent sur des vols nolisés.

Le gouvernement national et le transporteur national, Finnair, appuient largement le tourisme associé au Père Noël et la stratégie de marque. Visit Finland, notamment, dans sa brochure rédigée spécifiquement dans le but de faire le marketing du tourisme associé à la saison des fêtes, met l'accent sur l'origine supposée finlandaise du père Noël.

Why does the real Santa Claus live in Finland? You can meet him on any day of the year, without any charge, only in Finland. Santa Claus' own animal, the reindeer, lives in Finland. There is snow on the ground during the Christmas season. At the Arctic Circle, Santa Claus has a post office of his own and the world's only Main Santa Post Office. The Finnish Santa Claus receives by far the most letters from the children around the world. The only real amusement park of Santa Claus, the Santa Park, is situated in Finland. There are wide circles in Finland that have committed themselves to help Santa Claus. (Visit Finland, 2007)

Une fondation du père Noël a été créée en mars 2000 dans le but de conforter l'image de la Finlande à titre de résidence du père Noël.

The business idea of the Santa Claus Foundation is to develop and maintain world-wide the image of Finland as the real Christmasland and the home country of Santa Claus, and to provide opportunities for companies and other corporations to utilise this image.

The aim is, through concerted marketing activities, to build the Finnish Santa Claus brand into an added value factor to support the product and service marketing of the present (and future) partners. The Foundation will gather Finnish companies to support Santa Claus, and to benefit from the real, Finnish, Santa Claus. The operations of the Foundation is not the private business interest of any of the interested parties, it is for the good of the entire country of Finland, and its profits will be donated for charitable purposes.

Oy Joulupukin Maailma - Santa Claus World Ltd. is a company fully owned by the Foundation. Its purpose is the sale and supervision of the brand symbols of Santa Claus and the license rights and rights of use relating to the Santa Claus World Corporation. (Santa Claus Foundation, 2008)
En 2008 à Noël, il y avait 76 compagnies et organisations qui apparaissaient à la liste de la fondation (sur leur site Web) à titre de partenaires d'affaires. Néanmoins, malgré l'importance incontestée pour la région et le pays du tourisme associé au père Noël et son statut actuel de leader en termes du nombre de visiteurs, il y a des problèmes de long terme importants à régler en matière de promotion et de compétitivité de la destination.

\section{Défis liés à la compétitivité de la destination}

Les destinations de la Laponie elles-mêmes peuvent en arriver à se concurrencer de plus en plus pour être associées au père Noël. Bien que Rovaniemi soit le numéro un, l'augmentation de voyages internationaux vers d'autres destinations représente un défi important, surtout que des agents de voyages qui travaillent dans d'autres destinations laponnes tendent de plus en plus à promouvoir leurs forfaits des fêtes comme étant plus «authentiques». À long terme, le fait qu'il y ait en Laponie autant d'options associées au père Noël disponibles peut même soulever des questions quant au caractère unique de l'expérience laponne (Activities Abroad, 2008). C'est peutêtre le voyagiste Scan Meridian qui a émis les commentaires les plus sévères à ce sujet :

A long time ago, we hit upon the idea of taking children to meet the real Father Christmas. And in Lapland we found exactly what our young and young at heart travellers wanted. Then as now, the secret lay in travelling to villages in the snows where traditional Finnish Lutheran customs are played out on a small scale. There, Father Christmas can service his sledges and reindeer against a background of snow covered forests. And teams of huskies can happily bark up all the wrong trees, whilst you enjoy snowmobile rides and experience the habits and habitats of the reindeer and the huskies.

Unfortunately (one might say) the idea caught on so well that the mass-market operators felt they had to come crashing into the act. However, some villages were not big enough for their masses, so they persuaded the inhabitants of Rovaniemi, the so-called capital of Finnish Lapland, Saariselkä and Levi to put on snow-dusted plastic replicas of the real thing with discos, bars and all the other home "comforts" the mass-market seems unable to do without. Such trips have all the magic of a visit to a shopping centre in Croydon after a freak fall of snow (with apologies to Croydon).

But such once-in-a-lifetime experiences, we feel, need to be just that-genuine, traditional, memorable, mystical and exciting. We offer tobogganing and snowmobiling, not discos; ice-fishing, not fast food; pine clad rooms or open-fired log cabins, not soulless cell blocks; and herds of huskies, not people.

To avoid the masses, we've retreated to just three special places, Harriniva, Ylläshumina and Kakslauttanen. We have departures... for no more than 50 people at a time. If you would like to join us, we would love to have you but please remember: no discothèques, no fruit machines and no television. Just a family Christmas or New Year with like-minded people. (ScanMeridian, 2006) 


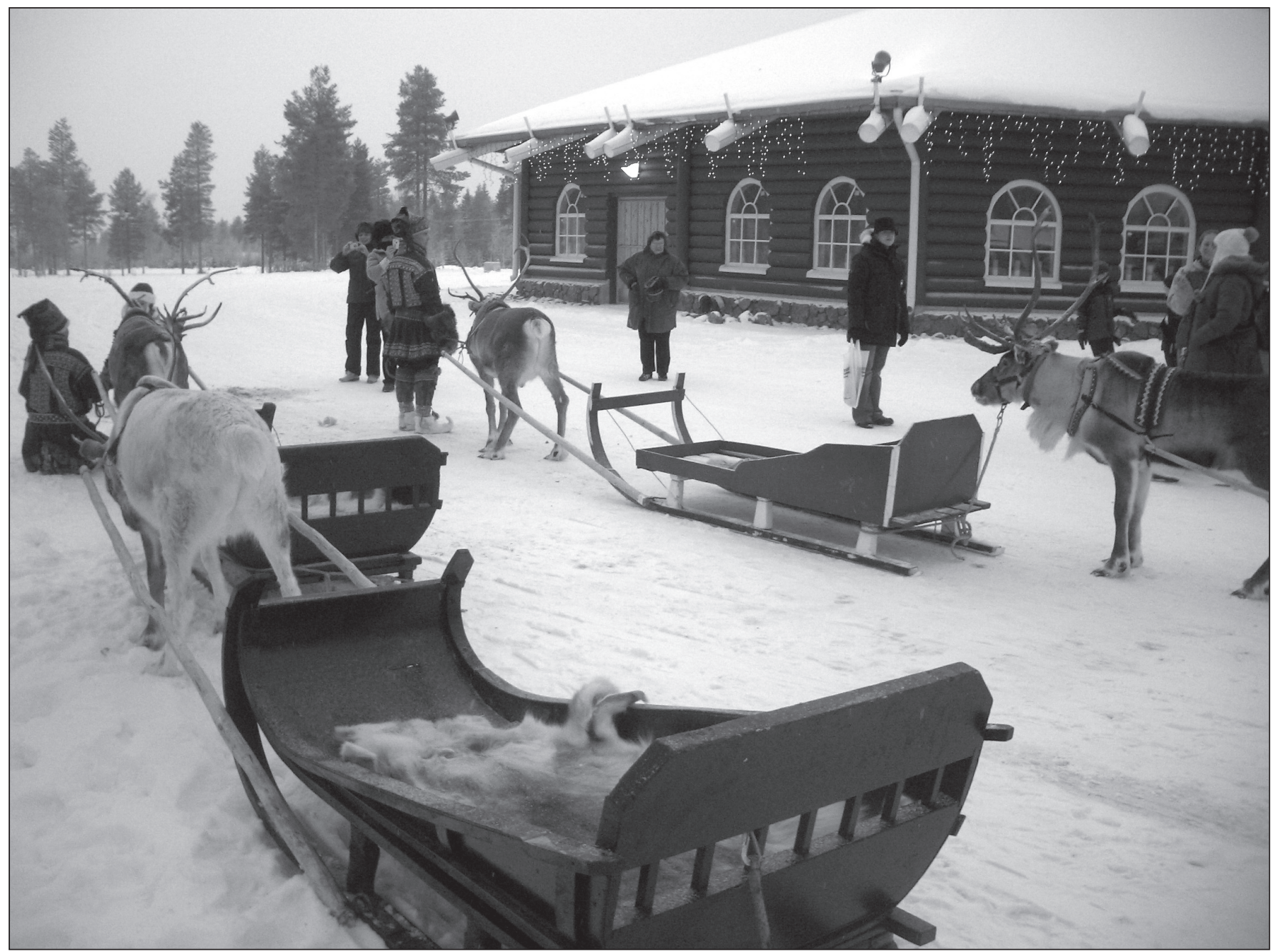

ILLUSTRATION 2 : Traineaux à rennes au Village du père Noël à Rovaniemi, en Finlande (photo : C. Michael Hall).

De même, en plus de vivre une concurrence accrue de son produit, Rovaniemi se différencie de moins en moins en termes d'image, même à l'intérieur de la Finlande. Quoique Rovaniemi soit la ville de Noël et la capitale de Noël, Turku se présente comme la ville finlandaise de Noël et Helsinki a maintenant sa rue Noël à Aleksanterinkatu ainsi que son marché St. Thomas Christmas. L'authenticité est notamment considérée comme partie intégrante de l'expérience de Noël de Turku. Sean Dodson (2007), dans le quotidien The Guardian, recommande ainsi à ses lecteurs : "So unless you want to entertain small children, avoid Lapland this Christmas and head for a weekend in old Finland instead. Go to the city that's so good they named it a capital twice.» Bien que les deux villes se positionnent à l'évidence sur des marchés différents, leur stratégie marketing soulève la grande question de la concurrence internationale des destinations de Noël.

En Alaska, la ville de North Pole près de Fairbanks cherche aussi à développer son volet Noël grâce à un concept de ville à thème et sa promotion reçoit de plus en plus de couverture internationale (voir entre autres Rush, 2007). Le bureau de poste de la ville reçoit aussi une quantité considérable de courrier adressé au Père Noël, ce qui offre des occasions substantielles de promouvoir la ville. Étonnamment, le Canada, pays qui reçoit le plus de courrier pour le père Noël, n'a pas associé celui-ci à une stratégie spécifique d'image de la destination, mais promeut plutôt l'idée que le père Noël vit au pôle Nord. Le Groenland se présente aussi comme la résidence du Père Noël et sa capitale, Nuuk, a même revendiqué le père Noël en installant la plus grosse boîte postale du monde à l'extérieur du bureau de poste du père Noël au centre d'information touristique. Cependant, la capacité du Groenland à répondre aux lettres adressées au père Noël est sérieusement menacée à la suite des coupures dans les dépenses gouvernementales, ce qui n'aidera probablement pas le développement à long terme du tourisme associé au père Noël dans ce pays. Par contraste, le ministère finlandais du Commerce supporte financièrement les dépenses engagées pour répondre aux lettres adressées au père Noël en Rovaniemi (Smith et Bagenal, 2006), ce qui fait encore ressortir la relation entre le père Noël et l'image de la destination. 
Or, il y a maintenant de nouveaux éléments qui risquent d'influencer l'image du père Noël et le tourisme des régions nordiques. La marchandisation et l'authenticité perçue de l'expérience père Noël sont manifestement des questions qui influencent déjà les agences de voyages dans leurs choix de destinations (Hall, 2008). Cependant, assurer aux consommateurs que certaines des images de l'environnement qui ont été utilisées comme images de marque des latitudes boréales polaires et de Noël, la neige et les rennes (illustrations 2 et 3 ) notamment, seront encore disponibles pour «consommation» visuelle est certes un aspect qui peut avoir des répercussions majeures à long terme. De plus en plus, comme résultat des inquiétudes liées à la fonte des glaces de mer et de la calotte glaciaire ainsi qu'aux espèces iconiques comme le caribou, le renne et l'ours polaire, l'imaginaire collectif commence à faire le lien entre changement climatique et père Noël (Robison, 2007).

La Finlande s'inquiète des conséquences que peuvent avoir les dangers liés à la neige sur la saison touristique de Noël, où Rovaniemi est dépeinte comme une destination potentiellement plus risquée que d'autres secteurs de villégiature. Par exemple, une histoire de l'Agence France-Presse concernant les conséquences du changement climatique sur la Laponie et le relatif avantage du climat d'Enontekioe sur Rovaniemi (Branchereau, 2007) a été couverte abondamment et internationalement. Au moins deux agences de voyages garantissent le «couvert de neige» dans leur publicité, dont Canterbury Travel (2008) qui déclare sur sa page Internet de FAQ (foire aux questions) :

Will there be snow?

A strange question to ask perhaps, but in the year 2003 one location in Lapland, namely Rovaniemi City, and its immediate environs, initially suffered from lack of snow. Following this occurrence Canterbury Travel transferred passengers on our day tours to a northern location where there was plenty of snow. As a result we have decided not to feature day tours to this immediate area. We still operate 2 one-day tours via Rovaniemi Airport (namely Christmas Past Christmas Present and the North Pole Post Office) to locations where snow levels were not a problem.

De même, Santadays (2008), agence qui se dit spécialiste de la Laponie, avance, en ce qui concerne la présence de la neige : "There will be snow in Lapland! Reports of lack of snow were exaggerated in previous years and only applied to a small area near Rovaniemi. The better quality trips take place in the far north of Lapland where snow is virtually guaranteed and counted in metres not inches! » Il reste néanmoins d'importantes questions de long terme à régler.

Des pronostics de changement climatique pour la Finlande laissent entendre que l'hiver est la saison qui subira les pires conséquences (Jylhä et al., 2008; 2004). On prévoit un réchauffement des hivers de 1,2 à 5 degrés Celsius pour les décennies à venir (de 0,6 à 1,6 degré Celsius pendant l'été en comparaison). Le réchauffement aura aussi comme effet de réduire le nombre de jours avec gel (où la température est sous zéro degré Celsius) et d'augmenter celui où la température sera au-dessus de zéro. D'ici la fin du siècle, on prévoit que la date du premier gel retardera de 15 à 30 jours comparé

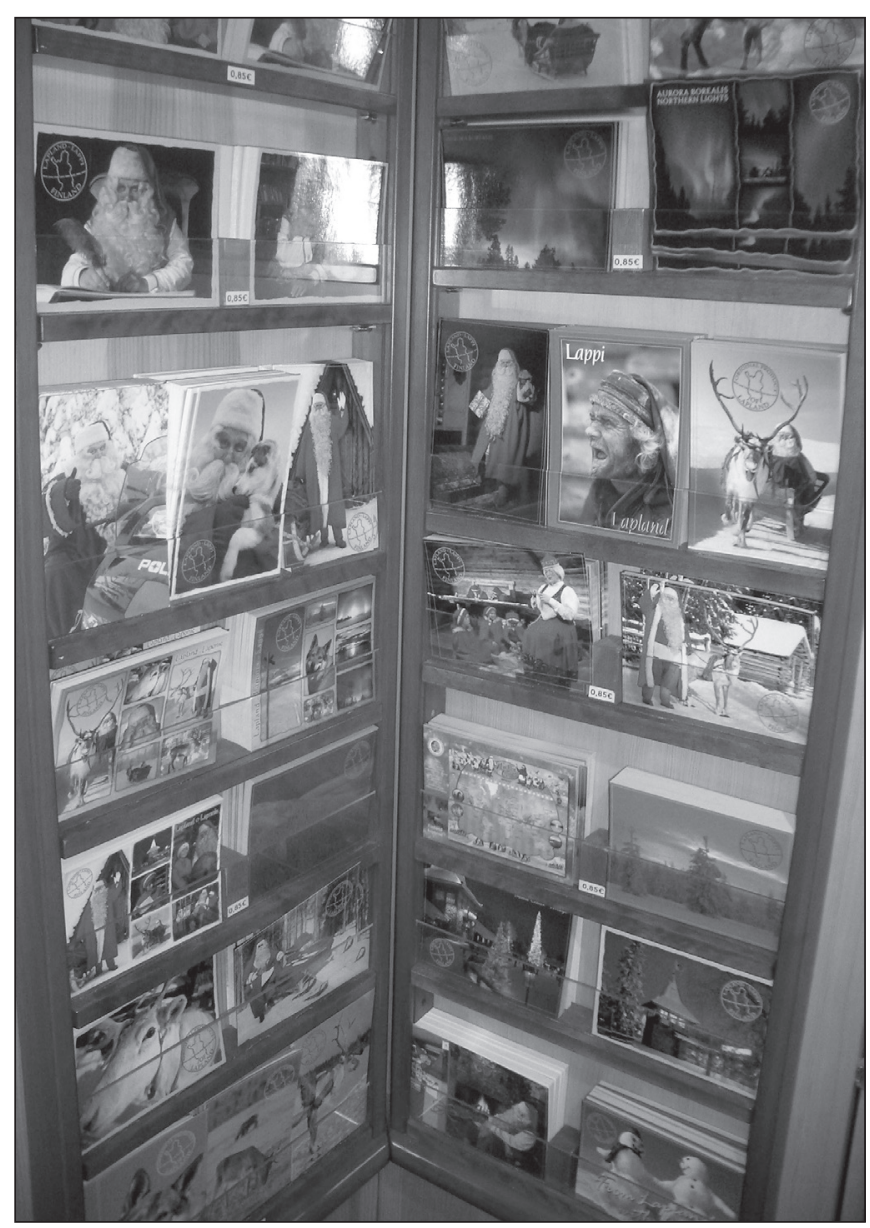

ILLUSTRATION 3 : Sélection de cartes de vœux des fêtes au Bureau de poste du père Noël, à Rovaniemi. Notons l'importance de la neige comme élément clé de l'imagerie du père Noël et de Noël (photo : C. Michael Hall).

à maintenant alors qu'on prédit que le dernier jour de gel arrivera de 10 à 30 jours plus tôt. Il n'y a pas de consensus quant au fait que le changement climatique puisse mener à la diminution du chiffre des extrêmes de températures en Finlande, mais on prévoit que la sévérité moyenne des jours avec gel à la grandeur de l'Europe du Nord s'atténuera (Jylhä et al., $2008: 451)$. On anticipe aussi que les précipitations hivernales augmenteront. À court terme, cela peut amener une augmentation des précipitations de neige dans le nord de la Finlande, mais à long terme on s'attend à ce que l'épaisseur du couvert de neige diminue partout. Le nombre de jours sans couvert de neige devrait aussi diminuer, mais relativement plus lentement en comparaison de la réduction de l'épaisseur de la neige. Selon Kirsti Jylhä et ses collaborateurs (2008: 452), on prévoit pour la fin du siècle des réductions de 40 à 60 jours des 240 jours d'enneigement annuel dans la partie la plus au nord de la Finlande.

Au volet des changements climatiques, les touristes devront aussi composer avec une plus grande variabilité des températures (Jylhä et al., 2008). C'est très inquiétant quand le mercure 
oscille continuellement autour du zéro. Un gel soudain après un réchauffement résulte souvent en une croûte de glace rigide qui empêche la pratique du ski de fond ou d'activités avec les chiens et les rennes, c'est-à-dire une part importante des activités touristiques d'hiver (Tervo, 2008), sans compter que les rennes ont peine à se nourrir à travers la croûte de glace. Le pronostic de survie à long terme du renne, un animal iconique et charismatique, n'est certes pas encourageant si l'on se fie aux recherches sur le changement climatique qui prédisent que les hivers seront plus doux et humides avec des chutes de neige plus abondantes, entraînant une diminution de la population de rennes (Lee et al., 2000).

Les chercheurs ont déjà commencé à s'intéresser aux conséquences potentielles du changement climatique sur les activités de tourisme hivernal en Finlande (Saarinen et Tervo, 2006; Tervo, 2008). Cependant, les répercussions à long terme sur l'image de la destination et la concurrence doivent encore être examinées, spécialement puisque la période de novembre-décembre est plus susceptible d'être affectée par le changement climatique que janvier-février. On a beaucoup mis l'accent sur la promotion de l'image de Noël, mais les effets potentiels du manque de neige pendant la période de Noël ne sont pas seulement l'obligation d'offrir des activités alternatives, mais il faut aussi être à la hauteur de son image de marque.

\section{Discussion et conclusions}

Le marketing et l'image de la destination constituent un processus graduel qui n'offre aucune garantie de succès pérenne pour différencier une destination dans l'esprit des consommateurs. Jusqu'à maintenant, certaines destinations, particulièrement en Laponie finlandaise, ont obtenu un succès substantiel en associant leur image au père Noël et à Noël afin de créer une forme d'avantage construit. Cependant, pour que la marque de la destination ait du succès à long terme, elle doit être crédible et refléter les atouts et les caractéristiques locaux. Malheureusement, pour un certain nombre de destinations nordiques qui cherchent à utiliser le mythe du père Noël comme principal avantage construit, une concurrence accrue en raison de toutes les destinations qui veulent s'associer à Noël et au père Noël lui-même soulève des doutes quant à l'authenticité qui a été imprimée dans l'esprit des consommateurs. Dans une telle situation, l'«avantage du premier» peut ne pas avoir de valeur à long terme puisque le fossé s'agrandit de plus en plus entre l'image de la marque et les valeurs sur lesquelles elle s'appuie.

Dans le cas de la Laponie, l'impact du changement climatique représente un autre défi à long terme pour l'image du pays de Noël et de la résidence du père Noël, puisqu'il pourrait bien mener à la perte ou à des changements importants à l'environnement de Noël qui, pourtant, est le principal fondement de l'authenticité de la marque. La promotion de la Laponie et de la Finlande se fait avec des images de neige pendant la période de Noël et la neige, pour bien des enfants (et des adultes), fait partie intégrante d'un Noël authentique. Une telle situation pose donc des défis énormes pour Rovaneimi qui est un point central des activités axées sur le père Noël; c'est aussi la ville pour laquelle cette association avec le père Noël a été la plus utilisée pour créer des opportunités touristiques. Sur les plans de l'image et de la compétition de la destination - heureusement pour la Laponie! -, peut-être que d'autres sites de haute latitude qui utilisent le père Noël comme moyen de différencier leur destination seront affectés par les changements climatiques et environnementaux (Arctic Climate Impacts Assessment 2005; Anisimov et al., 2007). Néanmoins, tel que déjà mentionné, les changements liés à la compétitivité des destinations découlant des changements climatiques ne se feront pas sentir seulement entre les pays, mais aussi entre les régions d'un même pays; dans le cas de la Finlande, il est bien évident que Rovaniemi sera de plus en plus en concurrence avec des destinations laponnes plus au nord qui sont peut-être mieux placées pour garantir de la neige pendant la saison de Noël.

L'utilisation du Père Noël comme moyen de différencier et de commercialiser la destination est une forme évidente d'avantage construit. Cependant, bien qu'un tel avantage soit construit socialement, surtout en ce qui constitue une authentique expérience du père Noël et de Noël, cet article a démontré que les éléments clés d'une image placée à l'avant-plan, la neige par exemple (tant sur le sol que celle qui tombe du ciel), les rennes ainsi que le paysage de fond - les conifères et la blancheur -, restent dépendants de l'environnement. Quand on promeut la résidence du père Noël, l'environnement est manifestement sous-jacent à la valeur réelle de l'image de la destination. Aussi, à long terme, peut-on s'attendre à ce que les changements climatiques et environnementaux aient des répercussions non seulement sur l'environnement physique et naturel et sur l'économie culturelle des régions nordiques, mais aussi sur la compétitivité relative des destinations.

Le père Noël sans la neige et sans ses rennes ne fait pas partie du pays de Noël imaginé par des millions de personnes dans le monde entier. Une telle situation constitue à l'évidence un défi majeur pour la Finlande et Rovaneimi, ainsi que d'autres destinations qui cherchent à promouvoir leur destination en utilisant un des mythes et des images polaires les plus puissants. Mais le message peut-être le plus fracassant, celui que le changement climatique risque de tuer le père Noël, peut engendrer une sensibilisation publique mondiale sur la menace que constitue le changement climatique pour l'environnement et les destinations polaires.

\section{Notes}

1 Une version antérieure de cet article a été rédigée pour la Swedish Polar Year Conference tenue à Umeå en octobre 2008.

\section{Bibliographie}

AALTONEN, Jarmo (2004), «Santa Claus Is a Strong Brand», Helsingin Sanomat, 15 décembre, [http://www.hs.fi/english/article/Santa+Claus+is +a+strong+brand/1101978059271], consulté le 1 1er avril 2008.

Activities Abroad The Activity Travel Company (2008), Santa Lapland Experience, [http://www.activitiesabroad.co.uk/Booking/ ViewByCategory.aspx?parentId=2\&categoryId=7], consulté le $1^{\text {er }}$ mai 2008.

American Marketing Association Marketingpower (2008), Dictionary, [http://www.marketingpower.com/_layouts/Dictionary.aspx], consulté le $1^{\text {er }}$ avril 2008. 
ANISIMOV, Oleg A., David G. VAUGHN, Terry CALLAGHAN, Christopher FURGAL, Harvey MARCHANT, Terry D. PROWSE, Hjalmar VILHJÀLMSSON et Johh E. WALSH (2007), «Polar Regions (Arctic and Antarctic)», dans Martin L. Parry, Osvaldo F. Canziani, Jean P. Palutikof, Paul J. van der Linden et Clair E. Hanson (dir.), Climate Change 2007: Impacts, Adaptation and Vulnerability, Cambridge, Cambridge University Press, p. 653-685.

Arctic Climate Impacts Assessment (2005), Impacts of a Warming Arctic: Arctic Climate Impacts Assessment, Cambridge, Cambridge University Press.

ARRUDA, William (2003), «Urban voice - Could Santa Be the World's Strongest Brand?», brandchannel.com, 22 décembre, [http://www.brandchannel.com/brand_speak.asp?bs_id=74], consulté le 1er avril 2008.

BRANCHEREAU, Gael (2007), "Global Warming May Soon See Santa Don Shorts", AFP Press Release, December 17.

BRISTOW, Gillian (2005), «'Everyone’s a Winner’: Problematising the Discourse of Regional Competitiveness ", Journal of Economic Geography, vol. 5, no 3, p. 285-304.

Canada Post (2007), Santa's Post Office Prepares for a Busy Season... Guinness World Records ${ }^{T M}$ Holder Gets Ready To Deliver!, communiqué de presse, 9 novembre, [http://www.canadapost.ca/personal/corporate/about/ newsroom/pr/archive-e.asp?prid=1260], consulté le $1^{\text {er }}$ avril 2008.

Canterbury Travel (2008), FAQ, [http://www.santa-holidays.com/html/faq. html], consulté le $1^{\text {er }}$ avril 2008.

Cards For Good Causes (2007), Christmas Card Facts and Figures, Information Sheet, Andover, Cards For Good Causes.

CLARKE, Peter (2006), «Christmas Gift Giving Involvement », Journal of Consumer Marketing, vol. 23, n ${ }^{\circ}$, p. 283-291.

CLARKE, Peter (2007), «A Measure for Christmas Spirit», Journal of Consumer Marketing, vol. 24, nº 1, p. 8-17.

COCA-STEFANIAK, J. Andres, Fabrizio STASI, Giovanna CODATO, Elena FRANCO et Gareth ROBERTS (2008), « Reclaiming Customers Through a Retailer-led TCM Scheme in Italy», Journal of Place Management and Development, vol. 1, no 1, p. 115-124.

COOKE, Philip et Loet LEYDESDORFF (2006), « Regional Development in the Knowledge-based Economy: The Construction of Advantage», Journal of Technology Transfer, vol. 31, n 1, p. 5-15.

DODSON, Sean (2007), «Christmas's Second City. Lapland Might Be the Capital of Christmas, but Finns Head to their Country's Oldest City of Turku for a More Traditional Festive Break», The Guardian, 18 décembre, [http://www.guardian.co.uk/travel/2007/dec/18/finland.christmasnewyear], consulté le $1^{\text {er }}$ avril 2008.

HALL, C. Michael (1997), «Geography, Marketing and the Selling of Places», Journal of Travel and Tourism Marketing, vol. 6, $\mathrm{n}^{\text {os }} 3-4$, p. 61-84.

HALL, C. Michael (2000), «The Tourist and Economic Significance of Antarctic Travel in Australian and New Zealand Antarctic Gateway Cities", Tourism and Hospitality Research: The Surrey Quarterly Review, vol. 2, n 2, p. 157-169.

HALL, C. Michael (2005), Tourism. Rethinking the Social Science of Mobility, Harlow, Pearson Education.

HALL, C. Michael (2007), «Tourism and Regional Competitiveness », dans John Tribe (dir.), Advances in Tourism Research: New Directions, Challenges and Applications, Oxford, Elsevier, p. 217-230.

HALL, C. Michael (2008), «Santa Claus, Place Branding and Competition », Fennia, vol. 186, n 1, p. 59-67.

HALL, C. Michael et Allan WILLIAMS (2008), Tourism and Innovation, London, Routledge.

HALL, C. Michael, Dieter K. MÜLLER et Jarkko SAARINEN (2009), Nordic Tourism: Issues and Cases, Clevedon, Channel View.
HALPERN, Nigel (2008), «Lapland's Airports: Facilitating the Development of International Tourism in a Peripheral Region", Scandinavian Journal of Hospitality and Tourism, vol. 8, $\mathrm{n}^{\circ}$ 1, p. 25-47.

HUKKINEN, Janne, Hannu HEIKKINEN, Kaisa RAITIO et Ludger MULLER-WILLE (2006), «Dismantling the Barriers to Entrepreneurship in Reindeer Management in Finland», International Journal of Entrepreneurship and Small Business, vol. 3, n ${ }^{\circ}$ 6, p. 705-727.

HYTÖNEN, Johanna (2007), «My Name is Claus, Santa Claus», Blue Wings, décembre, p. 36-41.

JACOBSEN, Jens Kr Steen (2009), «Prominent Promontory: The Social Construction of North Cape», dans C. Michael Hall, Dieter K. Müller et Jarkko Saarinen, Nordic Tourism: Issues and Cases, Clevedon, Channel View, p. 30-33.

JANSSON, Johan et Dominic POWER (dir.), (2006), The Image of the CityUrban Branding as Constructed Capabilities in Nordic City Regions, Oslo, Nordic Innovation Centre.

JYLHÄ, Kirsti, Heikki TUOMENVIRTA et Kimmo RUOSTEENOJA (2004), Climate Change Projections for Finland during the $21^{\text {st }}$ Century», Boreal Environment Research, vol. 9, nº 2, p. 127-152.

JYLHÄ, Kirsti, Stefan FRONZEK, Heikki TUOMENVIRTA, Timothy R. CARTER et Kimmo RUOSTEENOJA (2008), «Changes in Frost, Snow and Baltic Sea Ice by the End of the Twenty-first Century Based on Climate Model Projections for Europe», Climatic Change, vol. 86, $\mathrm{n}^{\text {os }} 3-4$, p. 441-462.

KOTLER, Philip et David GERTNER (2002), "Country as Brand, Product, and Beyond: A Place Marketing and Brand Management Perspective», Journal of Brand Management, vol. 9, $\mathrm{n}^{\text {os }} 4-5$, p. 249-261.

KOTLER, Philip, Christer ASPLUND, Irving REIN et Donald HAIDER (1999), Marketing Places Europe, London, Pearson Education.

LEE, Susan E., Malcolm C. PRESS, John A. LEE, Tim INGOLD et Terhi KURTTILA (2000), «Regional Affects of Climate Change on Reindeer: a Case Study of the Muotkatunturi Region in Finnish Lapland», Polar Research, vol. 19, n 1, p. 99-105.

LÄHTEENMÄKI, Maria (2006), «From Reindeer Nomadism to Extreme Experiences: Economic Transitions in Finnish Lapland in the $19^{\text {th }}$ and $20^{\text {th }}$ Centuries ", International Journal of Entrepreneurship and Small Business, vol. 3, nº 6, p. 696-704.

MALECKI, Edward J. (2004), «Jockeying for Position: What It Means and Why It Matters to Regional Development Policy When Places Compete», Regional Studies, vol. 38, n 9, p. 1101-1120.

MÜLLER, Dieter K. et Bruno JANSSON (dir.) (2007), Tourism in Peripheries: Perspectives from the Far North and South, Wallingford, CAB International.

NYSETH, Torill et Brynhild GRANÅS (2007), Place Reinvention in the North. Dynamics and Governance Perspectives, Stockholm, Nordregio.

O'CASS, Aron et Peter CLARKE (2002), «Dear Santa, Do You Have my Brand? A Study of the Brand Requests, Awareness and Request Styles at Christmas Time», Journal of Consumer Behaviour, vol. 2, $\mathrm{n}^{\circ}$ 1, p. 37-53.

OTNES, Cele, Young Chan KIM et Kyungseung KIM (1994), «All I Want for Christmas: an Analysis of Children's Brand Requests to Santa Claus», The Journal of Popular Culture, vol. 27, nº 4, p. 183-194.

PAGE, Stephen J. et C. Michael HALL (2003), Managing Urban Tourism, Harlow, Pearson.

PINE, Karen J. et Avril S. NASH (2002), «Dear Santa: The Effects of Television Advertising on Young Children », International Journal of Behavioral Development, vol. 26, nº 6, p. 529-539.

PINE, Karen J., Penny WILSON et Avril A. NASH (2007), «The Relationship Between Television Advertising, Children's Viewing and their Requests 
to Father Christmas», Journal of Developmental and Behavioral Pediatrics, vol. 28, nº 6, p. 456-461.

PRETES, Michael (1995), «Postmodern Tourism: the Santa Claus Industry», Annals of Tourism Research, vol. 22, n 1, p. 1-15.

ROBISON, Peter (2007), «Save Santa Claus's Home from Melting, Seattle Mayor Urges Kids», Bloomberg, 30 novembre, [http://www.bloomberg. com/apps/news?pid=20601082\&sid=aA3eUTBQFEd0\&refer=Canada $]$, consulté le $1^{\text {er }}$ avril 2008.

RUSH, Paul (2007), «Santa's Alaskan Home», New Zealand Herald, Associated Press Release, 11 décembre.

SAARINEN, Jarkko et Kaarina TERVO (2006), «Perceptions and Adaptation Strategies of the Tourism Industry to Climate Change. The Case of Finnish Nature-based Tourism Entrepreneurs», International Journal of Innovation and Sustainable Development, vol. 1, n 3, p. 214-228.

Santa Claus Foundation (2008), Business Idea of the Santa Claus Foundation, [http://www.santaclausworld.fi/santa/business-idea.html], consulté le 18 janvier 2009.

Santadays (2008), About Lapland, [http://www.santadays.co.uk/Lapland/ About_Lapland.asp], consulté le $1^{\text {er }}$ avril 2008.

ScanMeridian (2006), Father Christmas Holidays in Lapland, [http://www. scanmeridian.co.uk/christmas/], consulté le $1^{\text {er }}$ avril 2008.

SLOCUM, Rachel (2004), «Polar Bears and Energy-efficient Lightbulbs: Strategies to Bring Climate Change Home», Environment and Planning D: Society and Space, vol. 22, n ${ }^{\circ} 3$, p. 413-438.

SMITH, Nicola et Flora BAGENAL (2006), «Santa is Sorry, but the Elf Post Is Down ", The Sunday Times, 26 novembre, [http://www.timesonline. co.uk/tol/news/world/article650069.ece], consulté le $1^{\text {er }}$ avril 2008.
SNELLMAN, Ritva Liisa (2006a), «Is Santa Claus Becoming an Overworked Seasonal Jack-of-all-trades? Does the Stripping Santa Meet Ministry Guidelines? Is the Brand in Danger?», Helsingin Sanomat International Edition - Culture, 26 décembre, [http://www.hs.fi/english/article/ bUPDATED+2612b+Is+Santa+Claus+becoming +an+overworked+seas onal+jack-of-all-trades+/1135223912098], consulté le 1er avril 2008.

SNELLMAN, Ritva Liisa (2006b), «Santa Tourism Superpower. Four Days in Lapland Can Cost as much as a Fortnight in the Caribbean, but It's Worth It", Helsingin Sanomat international Edition - Business and Finance, 19 décembre, [http://www.hs.fi/english/article/Santa\%20 Tourism\%20Superpower/1135223794225], consulté le 1 er avril 2008.

TERVO, Kaarina (2008), «The Operational and Regional Vulnerability of Winter Tourism to Climate Variability and Change: the Case of the Finnish Nature-based Tourism Entrepreneurs", Scandinavian Journal of Hospitality and Tourism, vol. 8, $\mathrm{n}^{\circ}$ 4, p. 317-322.

US Census Bureau (2005), «Facts for Figures. The Holiday Season», CB05-FF.19-2 (mis à jour), 19 décembre, [http://www.census.gov/ Press-Release/www/releases/archives/facts_for_features_special_ editions/005870.html], consulté le $1^{\text {er }}$ avril 2008.

Visit Finland (2007), «The ABC on Santa Claus», [http://www.visitfinland. com/w5/trade/index.nsf/(Pages)/The_ABC_on_Santa_Claus], consulté le $1^{\text {er }}$ avril 2008.

Visit Finland (2008), «Christmas in Finland - Official Travel and Tourism Guide», [http://www.visitfinland.com/W5/index.nsf/(Pages)/UK_ Christmas?opendocument\&np=G], consulté le $1^{\text {er }}$ avril 2008.

\section{www.teoros.uqam.ca Un outil pour vos recheches}

Profil

Éditions : courante et future Index des articles

Abonnements

Appel à textes

Colloques

Ressources et liens

Coordonnées

\section{Venez découvrir le résumé indexé de plus de 400 articles portant sur le tourisme!}

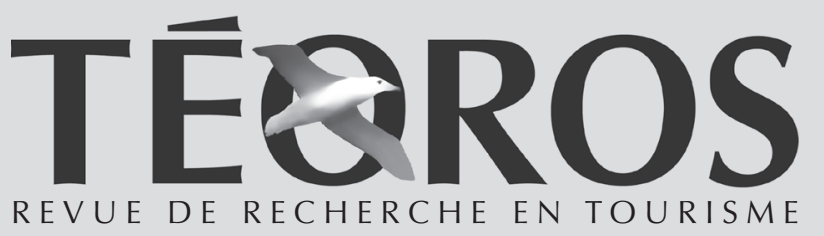

